\title{
Exploiting Location and Contextual Information to Develop a Comprehensive Framework for Proactive Handover in Heterogeneous Environments
}

\author{
G. Mapp, ${ }^{1}$ F. Katsriku, ${ }^{2}$ M. Aiash, ${ }^{1}$ N. Chinnam, ${ }^{1}$ R. Lopes, ${ }^{3}$ E. Moreira, ${ }^{3}$ \\ R. M. Porto Vanni, ${ }^{4}$ and M. Augusto ${ }^{5}$ \\ ${ }^{1}$ School of Engineering and Information Sciences, Middlesex University, Hendon, London NW4 4BT, UK \\ ${ }^{2}$ Department of Computer Science, University of Ghana, Accra, Ghana \\ ${ }^{3}$ Mathematics and Computer Sciences Institute, University of Sao Paulo, 13566-590 Sao Carlos, SP, Brazil \\ ${ }^{4}$ Department of Informatics, Federal Institute of Education, Science and Technology of Sao Paulo, 14801-600 Araraquara, SP, Brazil \\ ${ }^{5}$ Department of Information Systems, Santa Catarina State University, 89283-081 Sao Bento do Sul, SC, Brazil
}

Correspondence should be addressed to G. Mapp, g.mapp@mdx.ac.uk

Received 10 March 2012; Revised 7 June 2012; Accepted 8 June 2012

Academic Editor: Hoon Ko

Copyright () 2012 G. Mapp et al. This is an open access article distributed under the Creative Commons Attribution License, which permits unrestricted use, distribution, and reproduction in any medium, provided the original work is properly cited.

The development and deployment of several wireless and cellular networks mean that users will demand to be always connected as they move around. Mobile nodes will therefore have several interfaces and connections will be seamlessly switched among available networks using vertical handover techniques. Proactive handover mechanisms can be combined with the deployment of a number of location-based systems that provide location information to a very high degree of accuracy in different contexts. Furthermore, this new environment will also allow contextual information such as user profiles as well as the availability of using location and contextual information to provide efficient handover mechanisms. Using location-based techniques, it is possible to demonstrate that the Time Before Vertical Handover as well as the Network Dwell Time can be accurately estimated. These techniques are dependent on accurately estimating the handover radius. This paper investigates how location and context awareness can be used to estimate the best handover radius. The paper also explores how such techniques may be integrated into the Y-Comm architecture which is being used to explore the development of future mobile networks. Finally, the paper highlights the use of ontological techniques as a mechanism for specifying and prototyping such systems.

\section{Introduction}

The development and deployment of several wireless networks mean that mobile devices will have several wireless interfaces including 3G, WLAN, WiMAX, and LTE. This represents a significant development as users will want to be always connected from anywhere and at any time. This will be achieved using vertical handover techniques where connections will be seamlessly switched between available networks. As explained in [1], handover is defined as the changing of the network point of attachment (POA) of a mobile device. When the device moves to a new point of attachment which is technologically identical to the previous point of attachment, this is called horizontal handover. Vertical handover is defined as a handover where the new point of attachment comprises a different technology when compared with the previous point of attachment [2].

Proactive handover in which the mobile node actively attempts to decide when and where to handover can help to develop an efficient handover policy mechanism. This is because by using proactive handover, it is possible to minimize packet loss and service disruption as an impending handover can be signalled to the higher layers of the network protocol stack. However, in order to develop efficient proactive handover, location information and services are necessary. In addition, context-aware information based on user 
profiles, local network services, and information from other users can be used to ensure that the mobile user is always best connected or $(\mathrm{ABC})[3]$.

Information such as the position of Access Points, network coverage as well as the speed and direction of the mobile node can be used to develop equations which can calculate Time Before Vertical Handover or TBVH as well as the estimated Network Dwell Time or NDT in a given network. This paper develops a mathematical framework which can be used to calculate TBVH and NDT. Knowing TBVH and NDT allows mobile nodes to efficiently decide when is the best place to do a handover, such that there is very little handover latency or packet loss. It will be shown that this technique is dependent on having a good estimation of the handover radius which is, in turn, dependent on an accurate propagation model that needs to be dynamic and adaptable. This paper shows how contextual information about network conditions encountered by other mobile nodes could be used to build a more dynamic and adaptable propagation model.

Since it is not possible for mobile nodes to store the information on all the available networks, it is necessary that such information be available in the core network. This could be implemented as part of core network functionality for future mobile networks. Such an approach is being adopted as seen in the Media-Independent Information Service (MIIS) advocated by the IEEE 802.21 standards [4] which look at specifying Media Independent Handovers (MIH). However, this paper argues that in order to build a comprehensive platform, it is necessary to include information on users as well as on networking infrastructure.

This paper attempts to bring together several aspects of handover to form a coherent framework. It shows how these methods can be used to provide mobile nodes with effective handover policies and how such policies can be implemented on an architecture such as Y-Comm which is being used to build future mobile systems.

The unique contributions of this paper can be described as follows: using location information and an estimation of the handover radius, this work shows how TBVH and NDT are calculated. It shows how Media Independent Information Service (MIIS) can be extended to store location information on networking infrastructure. This work also shows how contextual information about the mobile node in terms of the signal strength needed to handover, as well as information about its previous journeys and other mobile nodes can be combined using Wireless Footprinting techniques. This contextual information can also be used to develop a more dynamic and adaptable propagation model. Finally, this paper shows how an ontology can be used as an implementation path to develop a prototype testbed. Compared to previous studies, this work presents a clear way forward for the development and deployment of proactive mechanisms in real mobile systems.

The rest of the paper is structured as follows: Section 2 discusses handover classification while Section 3 examines how handover coverage is specified. In Section 4 the mathematical framework is detailed while Section 5 examines the application of the mathematical framework to a specific network scenario. Section 6 looks at results for different handover radii. Section 7 investigates how the information on local networks can be acquired and stored. Section 8 looks at the Y-Comm Framework, particularly how Y-Comm deals with handover. Section 9 summarises previous work while the paper concludes with Section 10 which looks at conclusions and future work.

\section{Vertical Handover: A Detailed Classification}

In this section, we define the set of terms used in the context of handover. This presentation is divided into two broad types. The first has to do with a general handover classification which classifies handovers using general characteristics while the second is a more advanced classification dealing with the different mechanisms and inputs to do with handover.

2.1. General Handover Classification. Handovers can be classified in terms of when the connection to the previous POA is broken in relation to when the connection to the next POA is made, which device is in charge of handover, and the coverage areas of the relevant networks.

(i) Hard versus soft handover: in a hard handover, the connection to the previous POA is broken before the connection to the new POA is made. In soft handover, the connection to the next POA is made before the connection to the previous POA is broken.

(ii) Network-based versus Client-Based Handover: in network-based handover, the network makes the final handover decision while in client-based handover, it is the mobile device that decides when to handover. Though current cellular systems use network-based handovers, mechanisms such as Mobile IP [5] and Fast Mobile IP [6] are client-based. In fact, for heterogeneous environments client-based handover is favoured [7].

(iii) Upward versus downward Handover: where a network, say A, is completely covered by network B, then if we make a handover from network $A$ to network $\mathrm{B}$, this is referred to as an upward handover because we are going from a smaller network with substantial bandwidth to a network of a much larger coverage with lower bandwidth. While a handover from network B to network A is referred to as a downward handover because we are going from a larger to a smaller network.

2.2. Advanced Classification. An advanced type of handover classification is shown in Figure 1. Handovers can also be divided into two advanced types. Imperative handovers occur due to technological reasons only. Hence the mobile node changes its network attachment because it has determined by technical analysis that it is good to do so. This could be based on parameters such as signal strength, coverage, and the quality-of-service offered by the new network. These handovers are imperative because there may be a severe 


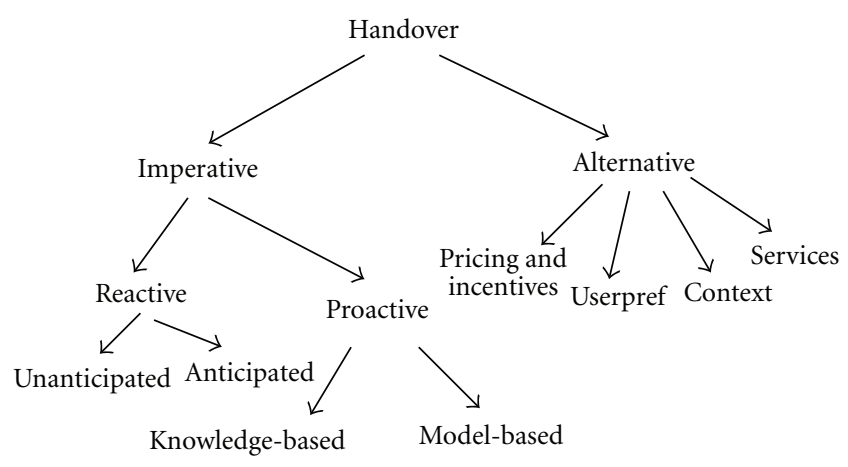

FIgure 1: Handover classification.

loss of performance or loss of connection if they are not performed. In contrast, alternative handovers occur due to reasons other than technical issues [8]. Hence there is no severe loss of performance or loss of connection if an alternative handover does not occur. The factors for performing an alternative handover include a preference for a given network based on price or incentives. User preferences based on features or promotions as well as contextual issues might also cause handover. Finally, there may be other network services that are being offered by certain networks.

Imperative handovers are, in turn, divided into two types. The first is called reactive handover. This responds to changes in the low-level wireless interfaces as to the availability or nonavailability of certain networks. Reactive handovers can be further divided into anticipated and unanticipated handovers [9]. Anticipated handovers are soft handovers which describe the situation where there are alternative basestations to which the mobile node may handover. With unanticipated handover, the mobile node is heading out of range of the current POA and there is no other base-station to which to handover. These handovers are therefore examples of hard handovers.

The other type of imperative handover is called proactive handover. These handovers use soft handover techniques. Proactive handover policies attempt to know the condition of the various networks at a specific location before the mobile node reaches that location. Proactive policies allow mobile nodes to calculate the Time Before Vertical Handover (TBVH) which enables them to minimize packet loss and latency experienced during handovers. Presently, two types of proactive handovers are being developed. The first is knowledgebased and attempts to know, by measuring beforehand, the signal strengths of available wireless networks over a given area such as a city. This could involve physically driving around and taking these readings [10]. These measurements need to be taken in different seasonal contexts as the effects of foliage on wireless propagation are well known [11]. The second proactive policy is based on a mathematical model which calculates the point when vertical handover should occur and the time that the mobile would take to reach that point based on its velocity and direction [12]. The accuracy of this approach is dependent on various factors including location technology, the propagation model used, network topology, and specific environments, for example, whether

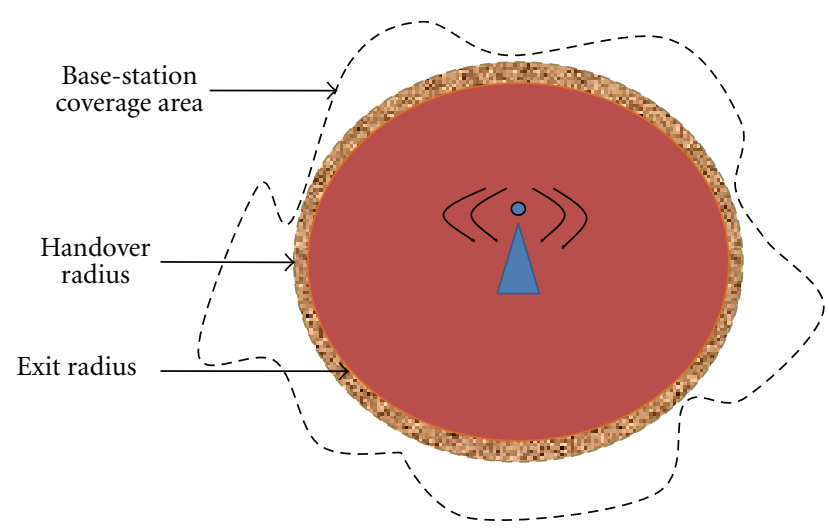

FIGURE 2: Network coverage.

the mobile node is indoor or outdoor [13]. In this paper, we will develop algorithms based on the model-based approach.

\section{Handover Coverage Parameters}

In this section, we introduce a set of network coverage terms. We first define the concept of a coverage area as the region where signals from a given POA can be detected. The coverage area is of an irregular shape. On the boundary of the coverage area signals from the POA are unreliable and beyond the coverage area, the signals from the POA cannot be detected. Since handover is a complex operation, it should be finished before the coverage boundary is reached. So we define a circle known as the handover threshold, and the corresponding radius is called the handover radius represented by $R_{H}$. Ideally, it is necessary to complete handover before or at the handover boundary to ensure smooth handover. In order to make sure that handover occurs at the handover boundary, we define another coverage area known as exit coverage represented by the exit radius or $R_{E}$. Handover must begin at the exit radius in order to ensure that it is completed at the handover boundary. The arrangement is shown in Figure 2 and discussed in [14]. The exit radius will therefore be dependent on the velocity, vel, of the mobile node. If we represent the time taken to execute a handover by $T_{E H}$, then we can say in

$$
T_{E H} \leq \frac{\left(R_{H}-R_{E}\right)}{\text { vel }}
$$

Hence

$$
R_{E} \leq R_{H}-\left(\operatorname{vel} * T_{E H}\right) .
$$

So the faster a mobile node moves the smaller the exit radius at which handover must begin. We will now continue our analysis using the handover radius. But given that we can find the handover radius and we know the velocity of the mobile node and the time taken to execute a handover, then we can calculate the exit radius.

3.1. Estimating Handover Radius Using Propagation Models. The accuracy of the system revealed above depends on a 
good estimation of the handover radius which, in turn, is dependent on the propagation models being used. Propagation models attempt to model the received signal strength of an electromagnetic signal at a given distance from where the signal is being transmitted. Propagation models are divided into 3 main types.

(i) Empirical Models. These models are based on mathematical equations that attempt to take into account the travelling effects on the electromagnetic signal [15]. Empirical models can further be divided into two categories: time dispersive and nontime dispersive models. Time dispersive models take into account channel characteristics such as multipath spread. Nontime dispersive models predict the path loss in terms of distance, height of antenna, and frequency. These models are mainly based on observations as well as measurements. Hata and Cost-231 are examples of nontime dispersive models.

(ii) Deterministic Models. These models are related to the propagation of electromagnetic waves to find out the strength of the received signal at the particular point. It requires a $3 \mathrm{D}$ view of electromagnetic waves. Ray tracing is an example of deterministic models.

(iii) Stochastic Models. These models generate predictions with the help of a series of random variables; these models are therefore less accurate than predictions based on empirical or deterministic models. But stochastic models require less information and have lower processing power requirements.

This paper concentrates on nontime dispersive empirical models.

3.2. Path Loss Models. In wireless communications there is some loss of signal strength between the transmitter and receiver. This is called path loss and is the essential parameter used to predict the strength of signals in radio systems at various locations. The path loss is also dependent on environmental conditions and will be different in rural, urban, and suburban environments. Path loss models are usually dependent on both the distance from the transmitter as well as the frequency of the signal being transmitted. We can calculate the path loss in all these environments with the help of path loss models. Path loss can therefore be used to find the handover radius.

3.2.1. Free Space Models. This model deals with path loss in free space. In this environment, the attenuation is proportional to the square of the distance between the transmitter and the receiver and also to the square of the frequency of the radio signal.

The Free Space Path Loss (FSPL) in decibels is given by

$$
\mathrm{FSPL}=20 \log _{10}(d)+20 \log _{10}(f)+32.44-G_{t}-G_{r}
$$

where $d$ is the distance from the antenna in kilometres, $f$ is the frequency of the signal in $\mathrm{MHz}, G_{t}$ is the gain at the transmitter, and $G_{r}$ is the gain at the receiver.
This formula is effective where multipath effects are minimal such as for satellites in space.

3.2.2. The Okumura Model. In urban and suburban areas, it is necessary to consider multipath effects. The Okumura model is used to model urban areas. The path loss (PL) is given by

$$
\mathrm{PL}=L_{f}+A_{\mathrm{mu}}-G_{\mathrm{ht}}-G_{\mathrm{hr}}-G_{\mathrm{area}},
$$

where $L_{f}$ is the free propagation path loss, $A_{\mathrm{mu}}$ is the attenuation of the medium relative to free space, $G_{\mathrm{ht}}$ is the gain due to the height of the base station antenna, $G_{\mathrm{hr}}$ is the gain due to height of the mobile node receiver, and $G_{\text {area }}$ is the gain due to the type of environment.

3.2.3. Hata Models. The Okumura model has been used as a base to evolve Hata models which can be deployed for urban and suburban environments.

For the urban areas,

$$
\begin{aligned}
L_{u}= & 69.55+26.16 \log _{10} f-13.82 \log _{10} h_{b}-C_{H} \\
& +\left(44.9-6.55 \log _{10} h_{b}\right) \log _{10} d,
\end{aligned}
$$

where $h_{b}$ is the height of the base station, $h_{m}$ is the height of the mobile node, and $C_{H}$ is the antenna height correction factor.

For small or medium cities,

$$
C_{H}=0.8+\left(1.1 \log _{10} f-0.7\right) h_{m}-1.56 \log _{10} f .
$$

For large cities, the formula is dependent on two frequency ranges.

So for $150 \leq f \leq 200 \mathrm{Mhz}$

$$
C_{H}=8.29\left(\log _{10}\left(1.54 h_{m}\right)\right)^{2}-1.1
$$

and for $200 \mathrm{MHz} \leq f \leq 1500 \mathrm{Mhz}$

$$
C_{H}=3.2\left(\log _{10}\left(11.75 h_{m}\right)\right)^{2}-4.97 .
$$

For Suburban areas,

$$
L_{s u}=L_{u}-2\left(\log _{10}\left(\frac{f}{28}\right)\right)^{2}-5.4
$$

where $L_{u}$ is the path loss in urban areas, $f$ is the transmission frequency in $\mathrm{MHz}$, and all path loss values are in $\mathrm{dB}$.

3.3. Using the Propagation Models. In order to find out the best handover radius we need the propagation characteristics as well as two other key parameters. The first is the power with which the signal is being transmitted. Hence the power of the signal at the transmitter is important as a more powerful signal would mean that a large handover radius is possible. The second factor is the signal strength threshold that is used to handover. Here, an absolute signal threshold could be used or a relative threshold could also be employed. 
Most Wireless LAN NIC cards can work between -70 and $-85 \mathrm{~dB}$ [16]. For WLANs, it has been decided to use the Free Space Model due to the high frequency being used. For cellular networks, Okumura and Hata models perform better than Free Space Models.

3.3.1. Network Interaction Classification. In order to build a complete framework, it is not only necessary to consider individual networks but also how individual networks in a given vicinity will interact with each other. This is even more important as there are completely different policies that are used for the deployment of different kinds of wireless networks. Cellular networks are rolled out on a national scale and issues of network coverage and user access are very carefully studied so that the deployment of masts and basestations will be done in a holistic way. In contrast, WiFi networks tend to be set up individually and in an ad-hoc manner. This means that we can consider the interaction of these networks in general. We do this by looking at the relationship between Network A and Network B. There are 3 cases to consider.

(i) The first is that Network A is completely covered by Network B. This occurs, for example, between a single local wireless LAN network and a colocated cellular network. In this context, mobile nodes moving in network $B$ and if, depending on their movement, they come into the range of network $\mathrm{A}$, then they would do an alternative downward handover to network A. After travelling through Network A, the mobile node would then do an imperative upward handover back to Network B.

(ii) The second network relationship occurs when Network A and Network B intersect. In this context, we need to understand the area of joint coverage and the implications for handover policy. If, given the mobile's direction, we can work out the distance for which the mobile node will be in contact with both networks, then we can work out the earliest and latest times at which a handover can occur.

(iii) The third network relationship is that Network A and Network B do not overlap at all. Hence the mobile node travelling between the two networks must do a hard handover when moving from Network A. It would shut down its connections, move to network $\mathrm{B}$ where it must contend for a channel, and then it would handover to the new channel and reopen its connections [17].

\section{The Mathematical Framework}

The Law of Cosines. In order to build a mathematical framework we need mathematical tools. An important tool is the law of cosines. The laws are given by the following equations.

$$
\begin{aligned}
& c^{2}=a^{2}+b^{2}-2 a b \cos \gamma, \\
& a^{2}=b^{2}+c^{2}-2 b c \cos \alpha, \\
& b^{2}=a^{2}+c^{2}-2 a c \cos \beta
\end{aligned}
$$

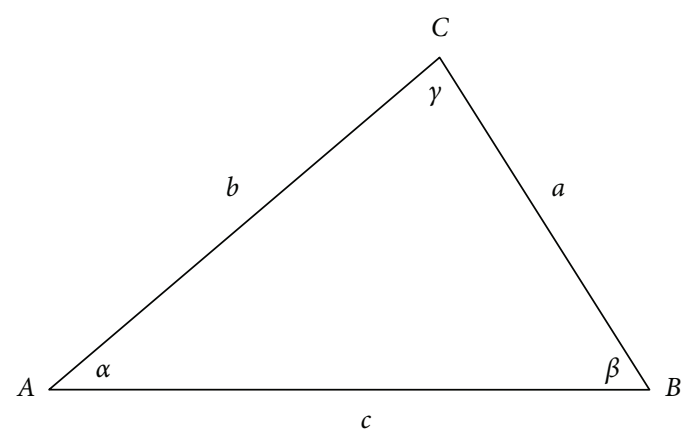

FIgURE 3: Law of cosines.

The sides and angles are shown in Figure 3. Further formulas can be derived, namely:

(i) the third side of a triangle if one knows two sides and the angle between them:

$$
c=\sqrt{\left(a^{2}+b^{2}-2 a b \cos \gamma\right)}
$$

(ii) the angles of a triangle if one knows the three sides:

$$
\gamma=\arccos \left(\frac{\left(a^{2}+b^{2}-c^{2}\right)}{2 a b}\right),
$$

(iii) the third side of a triangle if one knows two sides and an angle opposite to one of them:

$$
a=b \cos \gamma \pm \sqrt{\left(c^{2}-b^{2} \sin ^{2} \gamma\right)}
$$

4.1. Calculation for Different Handover Scenarios. In the section we look at different handover scenarios and calculate the Time Before Vertical Handover (TBVH) distance as well as the Network Dwell (ND) distance where appropriate. We now denote the handover radius by $R$.

4.1.1. Complete Coverage. For complete coverage we look at when Network A is completely covered by Network B, so Network A is a WLAN network and Network B is a cellular network such as GSM or UMTS. We now investigate upward handover as shown in Figure 4.

In this figure, $A$ is the centre of the WLAN, the mobile node is located at $C$, and knows its location using a GPS device. $R 1$ is the handover radius. The line $C F$ represents the present trajectory of mobile node. Since we know $d 1, R 1$, and angle $x$, as shown in [13], we can use (13) to obtain $C F$ as:

$$
C F=d 1 \cos x \pm \sqrt{\left(R 1^{2}-d 1^{2} \sin ^{2} x\right)}
$$

Hence $\mathrm{TBVH}=\mathrm{CF} / \mathrm{vel}$.

Next we consider downward handover, where the mobile node is going from the larger coverage network to a smaller network. This is shown in Figure 5.

The WLAN network is given by its radius $R 2$ and centre $B$. The mobile node is currently located at $C$ and is travelling 


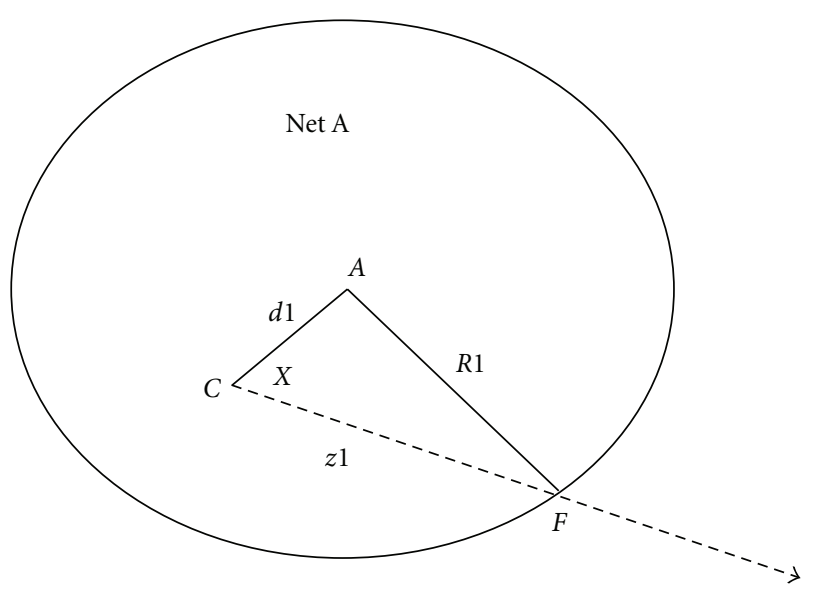

FIgURE 4: Upward handover.

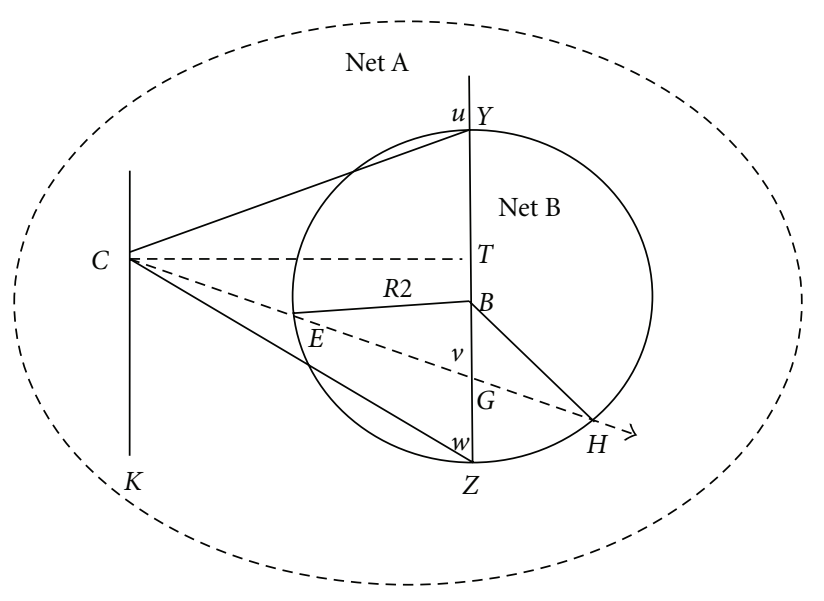

FIgURe 5: Downward handover.

on trajectory CEGH. The vertical axis of the WLAN is given by the points $Y$ and $Z$. These points can be easily obtained by the mobile node because it knows $R 2$ and $B$. From knowing $Y$ and $Z$ it is possible to calculate $C Y$ and $C Z$, respectively.

In terms of angles, we define them as relative to the vertical axis $Y Z$, so $C Y$ makes the angle $u$ with $Y Z$ while $C G$ makes the angle $v$ and $C Z$ makes the angle $w$ with $Y Z$. We can measure those angles directly from $C$ since the angle $u$ is $Y C K$, the angle $v$ is $G C K$, and the angle $w$ is ZCK. So the first thing we need to find out is if the trajectory of the mobile node means that it will or will not encounter the smaller network. We can easily verify this using angles $u, v$, and $w$. So we know that the mobile node will hit the WLAN because $u \geq v \geq w$. If $v>u$ or $v<w$ then the mobile node will not encounter the WLAN at all.

Since we can measure these angles and distances, we can now calculate $C G$. We use $C Y$ since

$$
\begin{gathered}
C T=C Y \sin (180-u)=C G \sin v, \\
C G=\frac{(C Y \sin (180-u))}{\sin v} .
\end{gathered}
$$

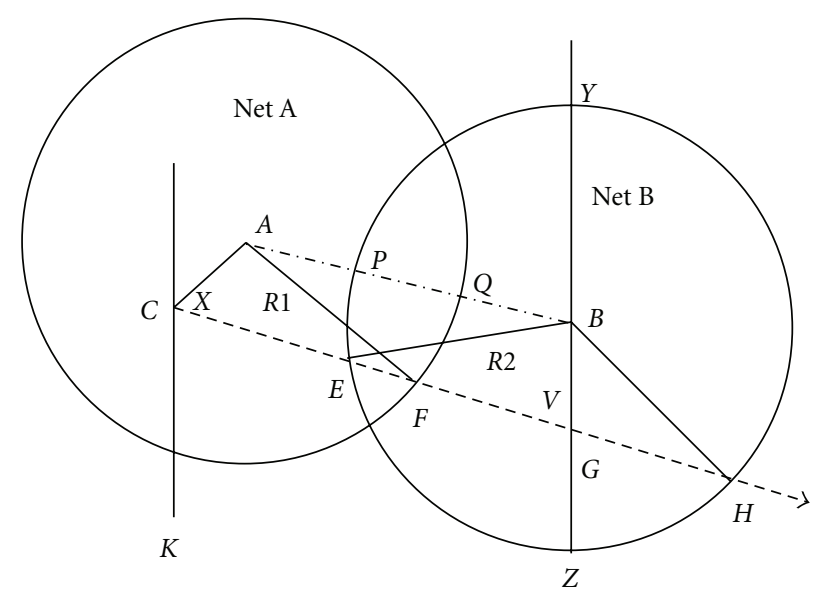

Figure 6: Intersecting networks.

So we know $C Z, C G$ and $C G Z$ is $(180-v)$; we can get GY from (13)

$$
G Z=C G \cos (180-v) \pm \sqrt{\left(C Z^{2}-C G^{2} \sin ^{2}(180-v)\right)} .
$$

Since $B G=B Z-G Z$ and $B Z=R 2$ then $B G=R 2-G Z$. So we can calculate $B G$. We know $B E=R 2, B G$, and angle $v$; hence

$$
E G=B G \cos v \pm \sqrt{\left(R 2^{2}-B G^{2} \sin ^{2} v\right)} .
$$

So the distance before handover is $C E=C G-E G$ and $\mathrm{TBVH}=C E /$ vel. We can also calculate the Network Dwell Time. To do this we need to calculate $G H$ we know that $B H=$ $R 2$ and the angle $B G H$ is $(180-v)$. So again using (13)

$$
G H=B G \cos (180-v) \pm \sqrt{\left(R 2^{2}-B G^{2} \sin ^{2}(180-v)\right)} .
$$

So the dwell distance of the mobile node in the WLAN if the mobile node continues in the same direction is $E H=E G+G H$. Hence, the estimated Network Dwell Time $(\mathrm{NDT})=E H / \mathrm{vel}$.

So we have examined the case of complete coverage.

4.2. Intersecting Networks. We next examine when the two networks intersect, this is shown in Figure 6.

To solve the intersecting networks scenario, we use the methods that we developed for upward and downward handovers. So we calculate $C F$ from (14), EG and $C E$ from (17), and $G H$ using (18). Using this information we can calculate the distance for which the mobile node will be moving in both networks. This is denoted by $E F=C F-C E$, where $C E=C G-E G$. This gives the mobile node the opportunity to do an alternative handover at $E$ or an imperative handover at $F$.

It also necessary to calculate the maximum joint coverage distance. This is given by $P Q$ and lies along the line between 


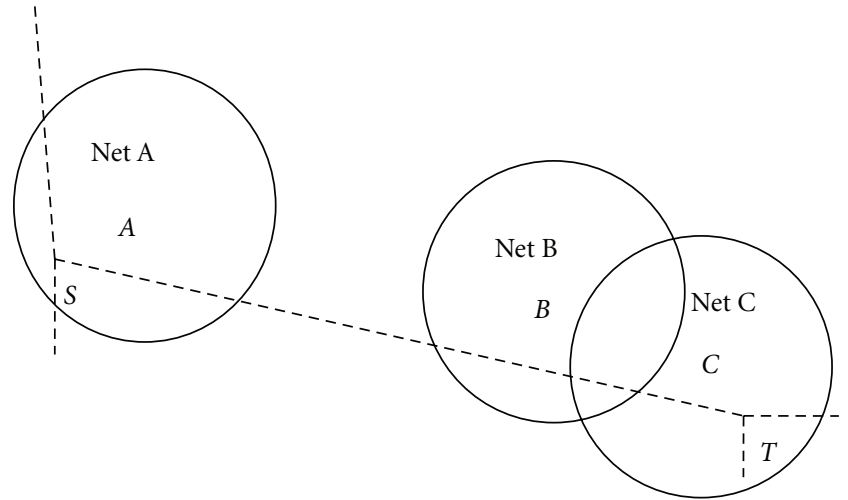

Figure 7: Applying the mathematical framework.

the two centres, $A B$. So $P Q$ is calculated as follows: $A Q=R 1$ and $A P=A B-R 2$

$$
\begin{gathered}
P Q=A Q-A P, \\
P Q=R 1-(A B-R 2), \\
P Q=R 1+R 2-A B .
\end{gathered}
$$

4.2.1. Nonintersecting Networks. For nonintersecting networks, we use (19). So the networks are nonintersecting when the distance between the two networks is greater than the sum of the radii of the two networks as expressed in (20)

$$
A B>R 1+R 2 \text {. }
$$

So given that we know the position of the Access Points and the handover radius for each network as well as the position, direction, and velocity of the mobile node, it is possible to estimate the TBVH for each network as well as the estimated dwell times in each network.

\section{Application of the Mathematical Framework}

In the section, we show how these formulas may be applied using a given scenario below. Let us suppose that the mobile node is going along a defined path. This is shown in Figure 7 and the entire region is also covered by a single cell of a cellular network such as GSM or UMTS.

There are two junctions $S$ and $T$ as well as three WLAN networks as shown. Network A provides access at Junction $S$ while Networks B and C are two other WLAN networks located along the $S T$ route. Since each network is a WLAN network, we will take the handover radius of 100 metres which is the accepted outdoor coverage of WLAN networks [18]. The coordinates of the key points are given in Table 1. C1 is the point on the path where the mobile node begins to calculate TBVH and its network dwell time in Network A.

Using these coordinates we can draw a detailed diagram of the upcoming calculations based on the mathematical framework detailed above. This is shown in Figure 8.

\subsection{Calculations for This Scenario}

5.1.1. Calculation for Network A. Important coordinates are: $Z 1=80,320, Y 1=280,320$.
TABLE 1: Coordinates at key points in metres.

\begin{tabular}{lcc}
\hline Symbol & $X$-cord & $Y$-cord \\
\hline$C 1$ & 40 & 480 \\
$S$ & 160 & 280 \\
$A$ & 180 & 320 \\
$B$ & 800 & 200 \\
$C$ & 960 & 160 \\
$T$ & 1000 & 120 \\
\hline
\end{tabular}

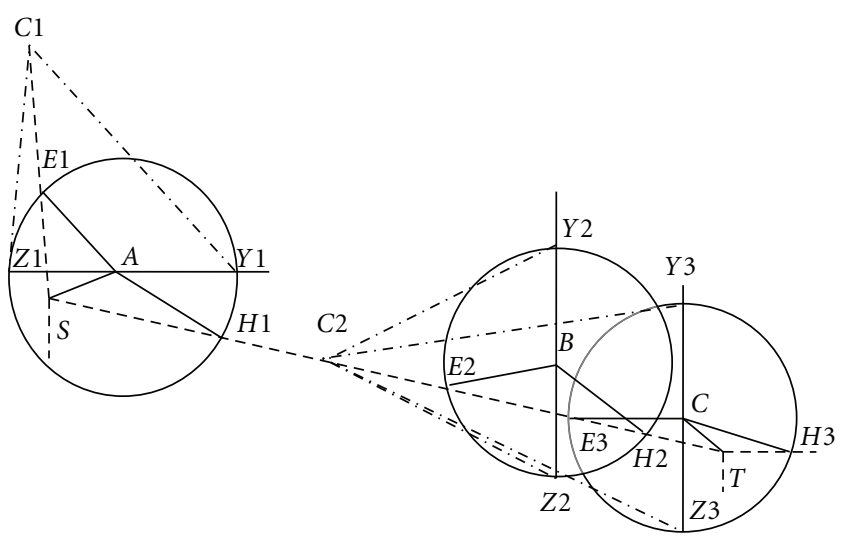

FIGURE 8: Analysis diagram.

The angle of $C 1$ to the vertical, $v$, is given by the gradient of $C 1 S$. However, we can get $G 1$ by simply using $C 1 S$ itself. So $G 1=x, 320$ where

$$
\frac{(x-40)}{(480-320)}=\frac{(160-40)}{(480-280)}
$$

so $G 1=(136,320)$, hence $A G 1=180-136=44$ and $v=$ $\arctan (120 / 200)$

$$
E 1 G 1=A G 1 \cos (90+v) \pm \sqrt{\left(R^{2}-A G 1^{2} \sin ^{2}(90+v)\right)}
$$

Note we must use the angle $(90+v)$ as we are using the horizontal axis, not the vertical one.

$E 1 G 1=69.97143$ and $C 1 G 1=186.59046$ and $C 1 S=$ 233.23808. So TBVH distance, $C 1 E 1=C 1 G 1-E 1 G 1=$ $116.61903 \mathrm{~m}$.

We find $S H 1$; we need to find the angle $A S H 1$. To find this we find the angle to the horizontal of $A S$ and the angle to the horizontal of $S T$ to capture the change of direction

$$
\begin{aligned}
\operatorname{ASH} 1=x= & \arctan \left(\frac{(320-280)}{(180-160)}\right) \\
& +\arctan \left(\frac{(280-120)}{(1000-160)}\right),
\end{aligned}
$$


TABLE 2: Results for Network A.

\begin{tabular}{lccc}
\hline Parameter & Symbol & Value $(\mathrm{m})$ & Handover \\
\hline TBVH distance & $C 1 E 1$ & 116.61903 & Downward \\
Network Dwell distance & $E 1 H 1$ & 219.04717 & Upward \\
\hline
\end{tabular}

$x=74.21925$ and $A S=44.72136$,

$$
S H 1=A S \cos (x) \pm \sqrt{\left(R^{2}-A S^{2} \sin ^{2}(x)\right)}
$$

$S H 1=102.42812, E 1 S=C 1 S-C 1 E 1=233.23808-$ $116.61903=116.61905, E 1 H 1=102.42812+116.61905=$ 219.04717.

The results for Network A are summarised in Table 2.

5.1.2. Calculation for Network B. We first consider $C 2$ which is an arbitrary point along ST. It is at $C 2$; the mobile node does calculations to determine the TBVH distance from $\mathrm{C} 2$, the expected time that will be spent in both networks and the distance that the mobile node will be under the coverage of the two networks. We have chosen the $x$-coordinate of $C 2$ to be 480 . Therefore the $y$-coordinate is calculated by using the gradient of ST. If $C 2=(480, y)$ then

$$
\frac{(480-160)}{(280-y)}=\frac{(1000-160)}{(280-120)} \text {. }
$$

Hence $C 2=(480,219.04762)$.

$v$, which is the angle of $S T$ to the vertical $=$ $\arctan ((1000-160) /(280-120))$.

In addition, since $G 2$ is along $S T$ if $G 2=(800, y)$, then

$$
\frac{(800-160)}{(280-y)}=\frac{(1000-160)}{(280-120)},
$$

$G 2=(800,158.09524)$.

Hence, $B G 2=200-158.09524=41.90476$,

$$
E 2 G 2=B G 2 \cos v \pm \sqrt{\left(R^{2}-B G 2^{2} \sin ^{2} v\right)}
$$

$E 2 G 2=98.97524$

We also calculate $\mathrm{G} 2 \mathrm{H} 2$

$$
\begin{aligned}
G 2 H 2= & B G 2 \cos (180-v) \\
& \pm \sqrt{\left(R^{2}-B G 2^{2} \sin ^{2}(180-v)\right)},
\end{aligned}
$$

$G 2 H 2=83.29346, C 2 G 2=325.75319$.

Hence, $C 2 E 2=C 2 G 2-E 2 G 2=226.77795, E 2 H 2=$ $E 2 G 2+G 2 H 2=182.2687$.

5.1.3. Calculations for Network $C$. We use the same technique as used for Network B to calculate E3G3.

So $G 3=(960,127.61905)$.

Hence, $C G 3=160-127.61905=32.38095$,

$$
E 3 G 3=C G 3 \cos v \pm \sqrt{\left(R^{2}-C G 3^{2} \sin ^{2} v\right)},
$$

$E 3 G 3=100.8649$.
TABLE 3: Results for Network B.

\begin{tabular}{lccc}
\hline Parameter & Symbol & Value $(\mathrm{m})$ & Handover \\
\hline TBVH distance & C2E2 & 226.77795 & Downward \\
Min Network Dwell distance & $E 2 E 3$ & 160.98697 & Horizontal \\
Max Network Dwell distance & $E 2 H 2$ & 182.2687 & Horizontal \\
\hline
\end{tabular}

TABLE 4: Results for Network C.

\begin{tabular}{lccc}
\hline Parameter & Symbol & Value $(\mathrm{m})$ & Handover \\
\hline Min Network Dwell distance & $\mathrm{H} 2 \mathrm{H3}$ & 171.95314 & Upward \\
Max Network Dwell distance & $E 3 H 3$ & 193.23487 & Upward \\
\hline
\end{tabular}

We first calculate G3T since we know the coordinates of G3 and $T$ :

$$
G 3 T=40.71916
$$

So we can find $C T$

$C T^{2}=G 3 T^{2}+C G 3^{2}-2 G 3 T * C G 3 \cos (180-v)$,

$C T=56.56969$.

We find the angle $C T H=(180-\arccos (40 / C T))$.

So we can now find $\mathrm{TH} 3$

$$
T H 3=C T \cos C T H \pm \sqrt{\left(R^{2}-C T^{2} \sin ^{2} C T H\right)},
$$

TH3 $=51.65081 \mathrm{~m}$.

We also would like to find the overlap distance between the two networks.

We can do so by finding $G 2 G 3$ which we can find directly because we know the coordinates of $G 2$ and $G 3$ :

$$
G 2 G 3=162.87663 .
$$

So $G 2 E 3=G 2 G 3-E 3 G 3=162.87663-100.8649=$ 62.01173 .

So the intersection distance $=G 2 H 2-G 2 E 3=$ $83.29346-62.01173=21.28173$.

Finally the Maximum Dwell distance in Network $\mathrm{C}=$ $E 3 H 3=E 3 G 3+G 3 T+T H 3=193.23487$.

The Minimum Dwell distance occurs if the mobile node delays handover until it has left Network B.

So Minimum Dwell distance in Network C $=193.23487$ $21.28173=171.95314$.

Similarly the Minimum Dwell distance in Network B is $182.2687-21.28173=160.98697$.

So the results of networks B and C are shown in Tables 3 and 4 .

The results for Network A, B, and C are summarised in Table 5.

5.2. Checking the Results. In order to check these results, it was decided to draw the whole system to scale using a large sheet of graph paper and hence the results above were compared with the values on the graph. A scale of $1 \mathrm{~mm}$ to $4 \mathrm{~m}$ was used. The results compared favourably with the graphical results. A computer program [19] was also used to 
TABLE 5: Results for Networks A, B, and C for $H R=100 \mathrm{~m}$.

\begin{tabular}{lcccc}
\hline Network & Parameter & Symbol & Value $(\mathrm{m})$ & Handover \\
\hline \multirow{2}{*}{$\mathrm{A}$} & TBVH dist & $C 1 E 1$ & 116.61903 & Downward \\
& ND dist & $E 1 H 1$ & 219.04717 & Upward \\
\hline$B \cap C$ & Intersect dist & $E 3 H 2$ & 21.28173 & Alternative \\
\hline \multirow{4}{*}{$\mathrm{B}$} & TBVH dist & $C 2 E 2$ & 226.77795 & Downward \\
& Min ND dist & $E 2 E 3$ & 160.98697 & Horizontal \\
& Max ND dist & $E 2 H 2$ & 182.26870 & Horizontal \\
\hline \multirow{2}{*}{$\mathrm{C}$} & Min ND dist & $H 2 H 3$ & 171.95314 & Upward \\
& Max ND dist & $E 3 H 3$ & 193.23487 & Upward \\
\hline
\end{tabular}

check these values. The program was done in C\# and read the location of the mobile node, the location of the different networks as well as the handover radius from a table in an SQL database and calculated the results for different points of the path as the mobile moved along the route. This verifies that this approach is viable. So the mobile node can calculate when and where to handover given that the mobile knows where the centre of each network is located and the handover radius of each network as well as the direction and speed of the mobile node [20].

\section{Results for Different Handover Radii}

6.1. Using Propagation Models to Get a More Accurate Handover Radius. As previously described, in order to get a more accurate estimation of the handover radius, we need to have the right propagation model as well as know the threshold value at which to handover. This is in fact dependent on the type of equipment being used and the power at the transmitter. To illustrate the issues, we imagine a scenario where the transmission power is 100 milliwatts, and the range of detection of the the WLAN receiver is chosen as $-82 \mathrm{~dB}$. Using the standard Free Space Model, the MATLAB tool was used to calculate the handover radius for this scenario. The result was a handover radius of 104 metres [21].

So we again calculate the values for the situation shown in Figure 8. The results are shown in Table 6. These results show that the TBVH times decrease while the Network Dwell distance and the intersect distance increase, with the latter increasing by over 40 percent. Thus small changes in the handover radius can affect handover decisions. This is also more important for 3G and UMTS systems where different propagation models will produce different results. For these systems, it is assumed that the mobile node will use a threshold of around $-120 \mathrm{~dB}$ [22]. The handover radius for urban and suburban Hata models are given in Table 7. These results again highlight the fact that if a wrong model is used then the values of TBVH and NDT would not be very accurate and therefore the proposed solution would not be useful.

These results show that in order to really build an efficient proactive handover system which can be managed in an autonomous manner, it is necessary to have more accurate information about the networking infrastructure in the local
TABLE 6: Results for Networks A, B, and C for HR = $104 \mathrm{~m}$.

\begin{tabular}{lcccc}
\hline Network & Parameter & Symbol & Value $(\mathrm{m})$ & Change $(\mathrm{m})$ \\
\hline \multirow{2}{*}{$\mathrm{A}$} & TBVH dist & $C 1 E 1$ & 112.3 & -4.38 \\
& ND dist & $E 1 H 1$ & 227.7 & 7.95 \\
\hline$B \cap C$ & Intersect dist & $E 3 H 2$ & 29.90 & 8.62 \\
\hline \multirow{3}{*}{$\mathrm{B}$} & TBVH dist & $C 2 E 2$ & 222.4 & -4.38 \\
& Min ND dist & $E 2 E 3$ & 161.1 & 0.11 \\
& Max ND dist & $E 2 H 2$ & 191.1 & 8.83 \\
\hline \multirow{2}{*}{$\mathrm{C}$} & Min ND dist & $H 2 H 3$ & 171.9 & -0.05 \\
& Max ND dist & $E 3 H 3$ & 201.8 & 8.57 \\
\hline
\end{tabular}

TABLE 7: Handover radius for 3G, UMTS at $-120 \mathrm{~dB}$.

\begin{tabular}{lc}
\hline Propagation model & Handover radius $(\mathrm{m})$ \\
\hline Urban Hata Model & 1850 \\
Suburban Hata Model & 1980 \\
\hline
\end{tabular}

area including the location of the Access Points/Base-stations as well as the power being transmitted at these locations plus a propagation model that represents the wireless network characteristics in that local area. Using this information, it would be possible to calculate the handover radius. This information along with the location and velocity of mobile node plus the $\mathrm{dB}$ thresholds for the wireless interfaces on the mobile device will make it possible to do effective vertical handover using the mathematical framework shown above.

\section{Information on Networks in the Local Area}

The approach presented shows that proactive handover can be performed over several networks that may be a few kilometres from the mobile node. In this context, it is not possible for the mobile node to deploy its network discovery mechanisms via its wireless network interfaces to find all the related networks. Hence information on networks in the local area needs to be stored in the core network.

7.1. Using IEEE 802.21. Such a facility has been defined in the IEEE 802.21 standards which specify Media Independent Handover (MIH) mechanisms that can be used to manage several wireless networks [23]. The MIH specifies 3 main mechanisms.

(i) Media-Independent Event Service or MIES: this service is used to relay events from low-level device interfaces to the upper layers via the MIH service layer. Applications register with the MIH layer the events in which they are interested. The MIH layer is, in turn, sent notification of events occurring at the lower level interfaces. Events include Link_Detected, Link_Up, Link_Down, Link_Going_Down, Link Hand-over_Imminent, and Link_Handover_Complete. The MIH layer then sends MIH event notifications to applications which want to be notified about specific events. 
(ii) Media-Independent Command Service or MICS: this allows applications to send commands to the lowlevel interfaces via the MIH layer. Applications can use MICS commands to find out the status of various interfaces. Commands sent to MIH layer by the upper layers include MIH_Link_Get_Parameters which gets the link status; the MIH_Link_Configure_Thresholds command is used to configure thresholds on mobile devices. Commands are also used to initiate handover to different target networks. Where appropriate, $\mathrm{MIH}$ commands are then converted to Link Commands which are sent to the relative network interfaces.

(iii) Media-Independent Information Service or MIIS: this service is used to allow mobile nodes to obtain network information within a geographical area so as to facilitate network selection and smooth handover.

7.1.1. Network Information Provided by the MIIS Service. Network information provided by the MIIS is divided into a number of containers. A higher level container can have lower level containers embedded within it. These are specified below.

(i) IE_CONTAINER_LIST_OF_CONTAINERS: at the highest level, there is a container for the list of heterogeneous networks in a given area.

(ii) IE_CONTAINER_NETWORK: each network is specified using a network container which gives all the information that depicts the access network including the number of POAs, IP subnet address ranges, and so forth.

(iii) IE_CONTAINER_POA: this contains all the information for a given POA.

The IE_CONTAINER_POA contains specific information on a given POA including its Media Access Control (MAC) address, Range of Channel, IP address, Geographical Location, System, and information on subnets. Some POA containers may also contain vendor-specific elements.

\subsection{A Prototype Database for Intelligent Proactive Handover.} It was decided to develop a prototype database on the networks in the local area [24] to allow mobile nodes to query a database server to get the required information that would allow them to calculate where and when is the best time to do the handover. The database was used to specify the WLAN networks in the local area. It also provided information on the parameters of the Free Space Model that had to be used in the local area, using the equation below

$$
\mathrm{RSS}_{p}=E_{t}-10 \beta \log _{10} l_{o p}+\xi
$$

where $\mathrm{RSS}_{p}$ is the received signal strength measured in $\mathrm{mW}$, $E_{t}$ is the transmission power of the AP in $\mathrm{mW}, \beta$ is the path loss component whose value is between 2 and $4, l_{o p}$ is the distance of the mobile node from the Access Point, and $\xi$ is the Gaussian distributed random variable.

Thus if this model is used, the values of $\beta$ and $\xi$ must be calculated for every local context and thus these values need
TABLE 8: Network information database.

\begin{tabular}{lc}
\hline Parameter in database & Data type of the field \\
\hline Latitude & double \\
Longitude & double \\
Channel number & int \\
Coverage radius & int \\
Transmit power & int \\
Mac address & Varchar $(50)$ \\
Path loss exponent & int \\
Gaussian distribution exponent & int \\
\hline
\end{tabular}

to be stored in a local database. The stored parameters are shown in Table 8.

The program was written in Java and showed that the mobile node could read the information from the database and then was able to calculate the handover radius, given the values of the $\beta$ and $\epsilon$. Based on these values it can then calculate TBVH and estimate NDT. It is able recalculate these values if the mobile node changes direction.

7.3. Determining the Exit Radius. In Section 3 we defined the exit radius as

$$
R_{E} \leq R_{H}-\left(\operatorname{vel} * T_{E H}\right) .
$$

Thus the exit radius, $R_{E}$, is very dependent on the handover radius, the velocity of the Mobile node, vel, and the time it takes to effect the handover, $T_{E H}$. The time taken to effect a handover was shown to be dependent by various factors as discussed in [25].

(i) The Detection Time, $t_{d}$. This is the time taken to detect the first signal of the new network. This can be done by detecting Router Advertisements or by the use of L2 triggers.

(ii) The Configuration Time, $t_{c}$. This is the time from detecting the network to the time taken by the mobile node to get and configure its Care-of-Address (COA).

(iii) The Registration Time, $t_{r}$. This is the time between the delivery of the Binding Update to the Home Agent and corresponding nodes and the time a packet arrives on the new interface.

(iv) The Adaptation Time, $t_{a}$. For vertical handovers, this is the time taken by the mobile node to adapt the connection to the new technology at the transport layer by adjusting the TCP state machine parameters (e.g., congestion window size, timeout timers, etc.), due to differences in the link characteristics.

For reactive handover, all four times must be added together since reactive handovers respond to network conditions as discussed in [11]. The mobile device therefore knows nothing beforehand about the characteristics of the various networks. This is not the case with an MIH-enabled device which can find out about networks via the MediaIndependent Information Service. For an MIH-enabled device, there is no detection time since the mobile node 
TABLE 9: Delay for proactive handover scenarios.

\begin{tabular}{lccccc}
\hline Source & Target & Handover & $t_{r}(\mathrm{~s})$ & $t_{a}(\mathrm{~s})$ & $T_{E H}(\mathrm{~s})$ \\
\hline 802.11g & HSDPA & Upward & 3 & 2 & 3 \\
HSDPA & $802.11 \mathrm{~g}$ & Downward & 4 & 0 & 4 \\
802.11g & $802.11 \mathrm{~g}$ & Horizontal & 4 & 0 & 4 \\
HSDPA & HSDPA & Horizontal & 1 & 0 & 1 \\
\hline
\end{tabular}

TABLE 10: Exit radius for different speeds in metres.

\begin{tabular}{lcccc}
\hline Network & Type & $T_{E H}(\mathrm{~s})$ & $R_{E} 5 \mathrm{Kmph}$ & $R_{E} 50 \mathrm{Kmph}$ \\
\hline A, C & Upward & 3 & 95.83 & 58.33 \\
B & Horizontal & 4 & 94.4 & 44.44 \\
\hline
\end{tabular}

would know where all the local networks are located. There is also a negligible delay for the configuration time since the mobile node will know the IP address of the target network. The Registration Time is still valid. In addition, for proactive networks the need for the transport protocol to adapt can be signalled before or during handover and not after the handovers occur. So it means that the adaptation time can be done in parallel with the registration time. So for proactive handover,

$$
T_{E H}=\operatorname{MAX}\left(t_{r}, t_{a}\right) .
$$

Using the measured results rounded up to the nearest second [11], in Table 9, we summarize the results for handover delay for proactive handovers between WLAN and $3 \mathrm{G}$ in our scenario.

Table 10 shows the exit radius for the different WLAN networks for a handover radius of 100 meters. We are interested in typical walking and driving speeds, so values of 5 Kilometres per hour (Kmph) and $50 \mathrm{Kmph}$ were selected. These results, which are given in metres, show that the exit radius is very dependent on velocity of the mobile node.

7.4. Using Community-Based Local Information Systems. In current networking environments, acquiring information about the local environment may be difficult for many reasons. As pointed out previously, cellular networks are carefully planned and detailed configuration characteristics may be regarded by some mobile operators as commercially sensitive information. WLAN systems may be set up on a perhome or per-person basis. This means that it is very unlikely that these networks would be registered with some central authority unless it is required by law.

Another way of gathering information about coverage and signal strengths is to allow users to access information on coverage data which was gathered from previous journeys along the same route or by other mobile nodes [26]. This has been explored in the concept of mobile virtual communities, which share aggregated data about mobile movement around particular nodes. The mechanism by which this data is stored is known as Wireless Footprinting.

Figure 9 shows a user with a Smart Phone walking through a number of wireless networks via a defined route/ path. There is a Wireless Footprint Server (WFS) which

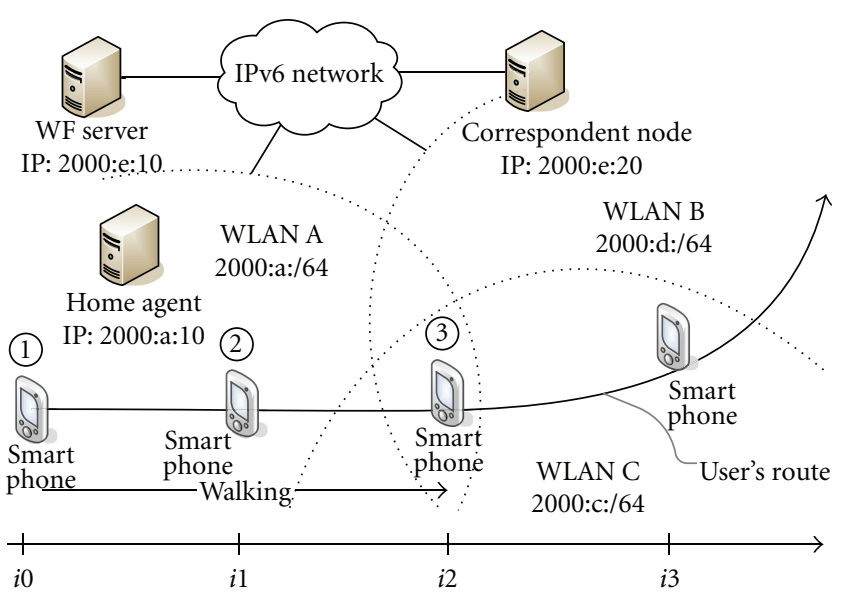

Figure 9: An example of Wireless Footprinting.

stores information about the wireless networks that the user has previously encountered at the present location. The Smart Phone contacts the WFS to find out if there is any wireless data associated with its current location. If so, it downloads the data and puts it in a local cache on the client node. The client can also ask for an aggregated summary of wireless characteristics in the local area based on the context information given to the server by other mobile nodes. The server therefore provides some key functions including:

(i) Storing client details: this mechanism allows the client to store details about wireless characteristics for various networks including signal strength, Access Point/Base-station IDs, bandwidth, QoS, and so forth, at a given location.

(ii) Context retrieval and storage: this allows the mobile node to retrieve context information about all the networks in the local area. This is similar to the MIIS service in IEEE 802.21.

(iii) Feedback search: this allows the server to provide information on wireless characteristics of local networks.

(iv) Cache consistency: the server pushes updates to the client so as to keep the local cache up-to-date.

(v) Handover history: the server can also record when and where the mobile node handed over. Though at times this may be useful, efficient handover depends on many real-time factors to characterize the state of the network.

It should be pointed out that a small experimental system [27] was built and the result showed improved connectivity during handover periods which indicates that better handover management was achieved.

7.5. Towards a Dynamic Propagation Model. The authors believe that the idea of community-based, context-aware information can also be used to develop more accurate and dynamic propagation models to characterize wireless characteristics in the local area. This is because the Wireless 
Footprinting Server or (WFS) can use the data on signal strengths at each location to build an accurate model. For WLAN this would mean finding the local parameters for the Free Space Model. For cellular networks the Hata models would be more accurately tuned. Thus using this approach an efficient proactive handover mechanism can be developed. Since the WFS is being updated frequently, it would be possible to calculate the most relevant propagation model in an adaptable and dynamic way which could take into account weather, and so forth. Ideally, therefore, it is necessary to have static information about the networking infrastructure as well as dynamic information which will be changing in real-time and can be used to make effective handover decisions.

7.6. Towards a Comprehensive Handover Framework. As shown above, contextual information can be used for effective imperative handover. However, the use of contextual information has also been used to look at alternative handover [28]. This means that the use of both location and contextual information along with the mechanisms presented above can be used to build a more comprehensive framework to manage vertical handover.

\section{The Need for a New Architecture for Mobile Networks}

This work above has shown that in order to build an efficient system, we will have to be able to integrate several new mechanisms and hence a framework is needed to do this. The main motivation for this is the belief that future mobile systems which will support heterogeneous networking must bring together mechanisms that support communication, mobility, QoS, and security in an integrated fashion. The YComm architecture is an architecture designed to address this goal. Y-Comm is based around a vision of the future predicated on two key assumptions: the first is that mobile devices will have several wireless interfaces and so must function in an increasing heterogeneous environment. The second is that the development and deployment of these wireless networks will point to a significant change in the architecture of the Internet. The Internet will no longer be represented by a single monolithic entity but by a superfast core composed of an optical backbone and fast access net-works while the end networks will be dominated by the deployment of wireless technologies. This is shown in Figure 10. Other network architectures for future mobile systems have been explored such as Ambient Networks [29] and Mobile Ethernet [30] but Y-Comm has been found to have a more open approach compared to these systems [31].

Y-Comm [32] therefore proposes two frameworks to represent this new reality. The first is called the Peripheral Framework and deals with operations and functions on the mobile node. The other framework is called the Core Framework and shows the functionality required in the core network to support the Peripheral Framework. The structure of the Y-Comm architecture is shown in Figure 11. A brief explanation of Y-Comm is now attempted starting with the

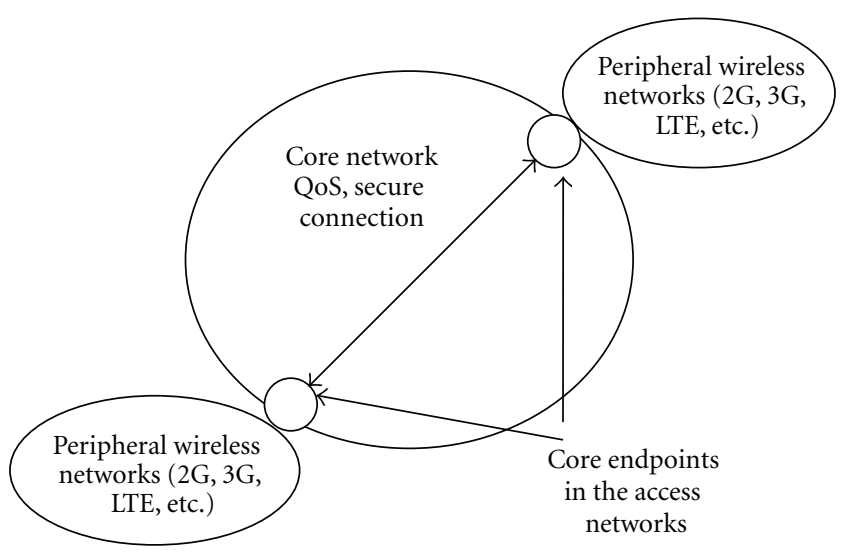

FIGURE 10: The future Internet structure.

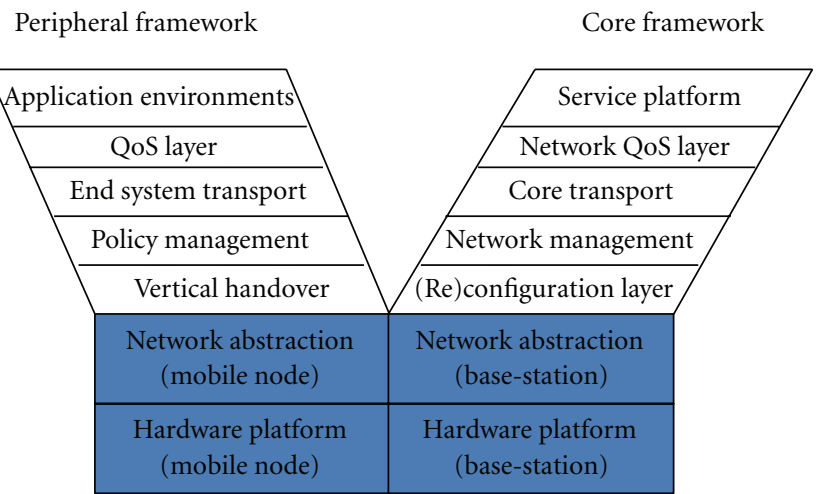

Figure 11: The Y-Comm framework.

lowest layer. A more detailed explanation can be found in $[7,33]$.

8.1. The Peripheral Framework. The Hardware Platform Layer (HPL) is used to classify all relevant wireless technologies. Hence different wireless technologies which are characterised by the electromagnetic spectrum, MAC, and modulation techniques make up this layer. The Network Abstraction Layer (NAL) provides a common interface to manage and control all the wireless networks. These first two layers for both frameworks are similar in functionality. In the Peripheral Framework, the Hardware Platform and the Network Abstraction layers run on the mobile to support various wireless network technologies while in the Core Framework these two layers are used to control the functions of base stations of different wireless technologies.

The Vertical Handover Layer (VHL) executes vertical handover. So this layer acquires the resources for handover, does the signalling, and context transfer for vertical handover. The Policy Management Layer (PML) decides whether and when handover should occur. This is done by looking at various parameters related to handover such as signal strength and using policy rules to decide both the time and place for doing the handover.

The End Transport Layer (ETL) is used to provide network and transport functions to the mobile nodes in 


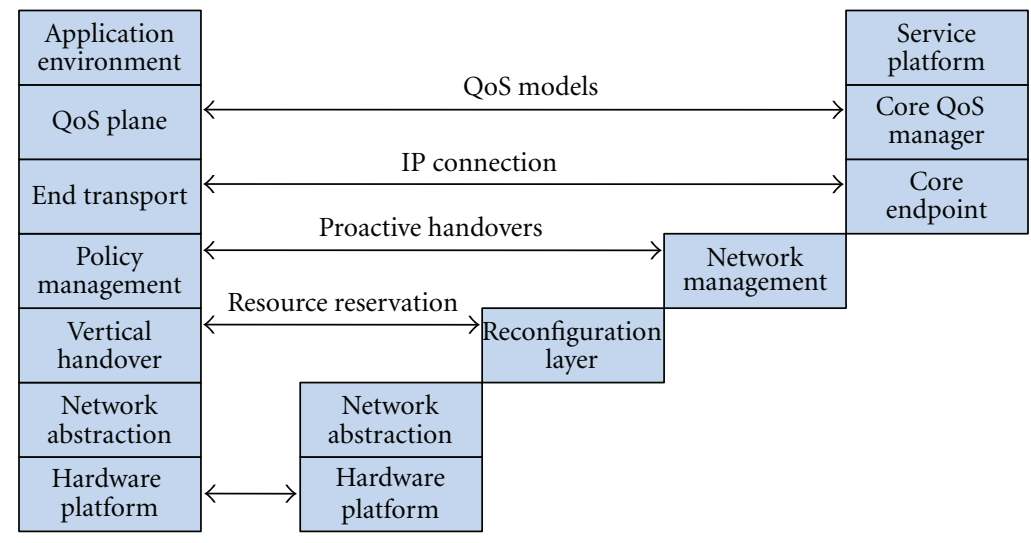

$\begin{array}{llll}\text { Mobile node } & \text { BS } & \text { RNC } & \text { 3G MSC/VLR } \\ \text { Core services }\end{array}$

FIgUre 12: Mapping of Y-Comm unto 3G/UMTS.

peripheral networks. It allows the mobile node to make endto-end connections across the core network. The QoS Layer (QL) in the Peripheral Framework supports two mechanisms for handling QoS. The first is defined as Downward QoS. This is where an application specifies its required quality-ofservice to the system and the system attempts to maintain this QoS over varying network channels. The other definition is Upward QoS where the application itself tries to adapt to the changing QoS. This layer also monitors the QoS used by the wireless network as a whole to ensure stable operation. The final layer of the Peripheral Framework is called the Applications Environments Layer (AEL). This layer specifies a set of objects, functions, and routines to build applications which make use of the framework.

8.2. The Core Framework. As previously mentioned, the first two layers of the Core Framework are engaged in controlling base-station operations. The third layer is called the Reconfiguration Layer (REL). It is a control plane to manage key infrastructure such as routers, switches, and other mobile network infrastructure using programmable networking techniques [34]. The Network Management Layer (NML) is a management plane that is used to control networking operations in the core. This layer can divide the core into a number of networks which are managed in an integrated fashion. It also gathers information on peripheral networks such that it can inform the Policy Management Layer running on mobile nodes about wireless networks at their various locations. In the context of the work already presented in this paper, the Wireless Footprinting Server (WFS) can be viewed as a manifestation of the Network Management Layer.

The next layer, called the Core Transport System (CTS), is concerned with moving data through the core network. Where the peripheral networks join the core network is called a core endpoint. Core endpoints are usually situated in access networks and several peripheral networks may be attached to a single-core endpoint. CTS is concerned primarily with moving data between core endpoints with a given QoS and a specified level of security.
The Network QoS Layer (NQL) is concerned with QoS issues within the core network especially at the interface between the core network and the peripheral networks. Finally the Service Platform Layer (SPL) allows services to be installed on various networks at the same time.

Though Y-Comm is a new architecture; it can be mapped onto current mobile infrastructure such as $3 \mathrm{G}$ or GSM as shown in Figure 12. As stated previously, the Peripheral Framework runs on the mobile node, while the Core Framework is distributed throughout the 3G/UMTS network infrastructure as shown.

Issues of security and quality-of-service have been investigated in the context of the Y-Comm architecture and new concepts such as Targeted Security Models have been developed $[35,36]$.

8.3. Proactive Handovers in Y-Comm. This section looks at proactive handovers in Y-Comm. This is shown in Figure 13. In order to perform vertical handover using a mathematical model approach, it is necessary to know the topology of these local networks. In Y-Comm, this information is managed by the Network Management Layer in the Core Framework. The mobile node therefore polls the NML to obtain information with regard to all local wireless networks, their topologies, and QoS characteristics. This information along with the direction and speed of the mobile as well as the QoS of ongoing connections is used by the Policy Management Layer to determine where and when handover should occur. The PML calculates TBVH-the period after which handover will occur as well as the estimated Network Dwell Time. This information is communicated to the Vertical Handover Layer which immediately requests resources to do a handover. Even though the resources are acquired early, handover actually takes place when TBVH expires.

In addition, once the PML decides to handover, the new IP address, the new QoS as well as TBVH, and estimated Network Dwell Time are communicated to the upper layers. Given TBVH, the upper layers are expected to take the necessary steps to avoid any packet loss, latency, or slow 
Peripheral network

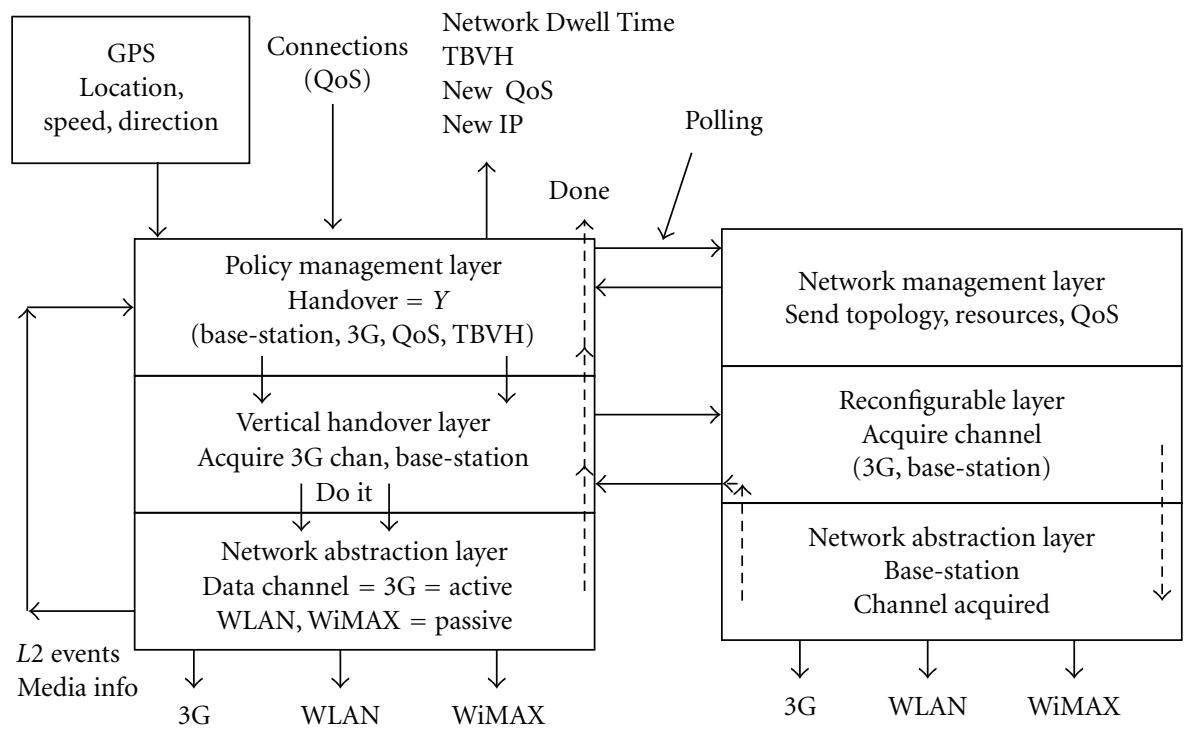

Figure 13: Proactive handover in the Y-Comm architecture.

adaptation. For example, it may be possible for the EndTransport Layer to signal an impending change in the QoS on current transport connections and to begin to buffer packets ahead of the handover. After handover, the previous channel used by the mobile node is released.

\subsection{Towards an Ontology for Proactive Handover in Y-Comm.} Ontologies are not only an elegant solution for modelling a problem. Ontologies are developed to formally describe semantic meanings of terms and their relationships in a domain. Heterogeneous entities working in a domain have semantic interoperability when they exchange the right information. Practices such as modularity, extensibility, and formal definitions of axioms can positively impact on the understanding and utilisation of systems mapped by ontologies. To accept the definitions of an ontology, explicitly or implicitly, means to be in agreement with the semantic representation of concepts and their relationships.

In order to develop a real system, it is necessary to define the functions and mechanisms in a way that will allow us to integrate different mechanisms and technologies. An ontology is a definition of a system with a detailed specification of each component. Ontologies will be used to describe the Y-Comm framework with a detailed specification of each component. The ontologies will assist in semantic interoperability between providers and users using Y-Comm. The definitions of the ontology will be stored in the ontology itself written in OWL (Web Ontology Language). The actual data will be stored in a database using the Resource Description Framework or RDF. However, the terms of the ontology are the descriptions of the data in the database [37].

An ontology for proactive in Y-Comm has been developed and is shown in Figure 14. It describes the relationships between different parts of the proactive handover system. Using this ontology, prototype code can be generated in Java showing how the functions for different parts of the lower layers in Y-Comm could be implemented. This is shown in Figure 15. Hence this approach represents a possible implementation path for future mobile networks.

\section{Previous Work}

Handover issues have been investigated for some time and it would be difficult to give a full summary of all the efforts. Original work was centred around horizontal handover in cellular networks and was done by mobile operators. With the introduction of Mobile IPv4 and Mobile IPv6 [5], client-based handover began to be investigated. For these mechanisms, handover latency is high because they only work at the network level as they are based on Router Advertisements (RAs) which are relatively slow. Fast Mobile IPv6 (FMIPv6) [6] was developed to make use of L2 events and triggers to reduce handover latency.

Reactive handover was studied in a number of simulations and in the Cambridge Wireless Testbed [38]. The testbed used the Vodafone 3G experimental network, with home and foreign WLANs and a wired IPv6 LAN. Using the testbed, PROTON [39], a policy manager for reactive handovers was developed. PROTON was implemented using a 3-layer structure.

However, with the rise of heterogeneous networking there was more interest in handover prediction techniques. This was explored using three research methods.

(i) History-Based Prediction.

(ii) Coverage-Based Prediction.

(iii) Mathematical-Modelling-Based Prediction.

9.1. History-Based Prediction. This prediction is based on the past movements of the mobile device in the network. 


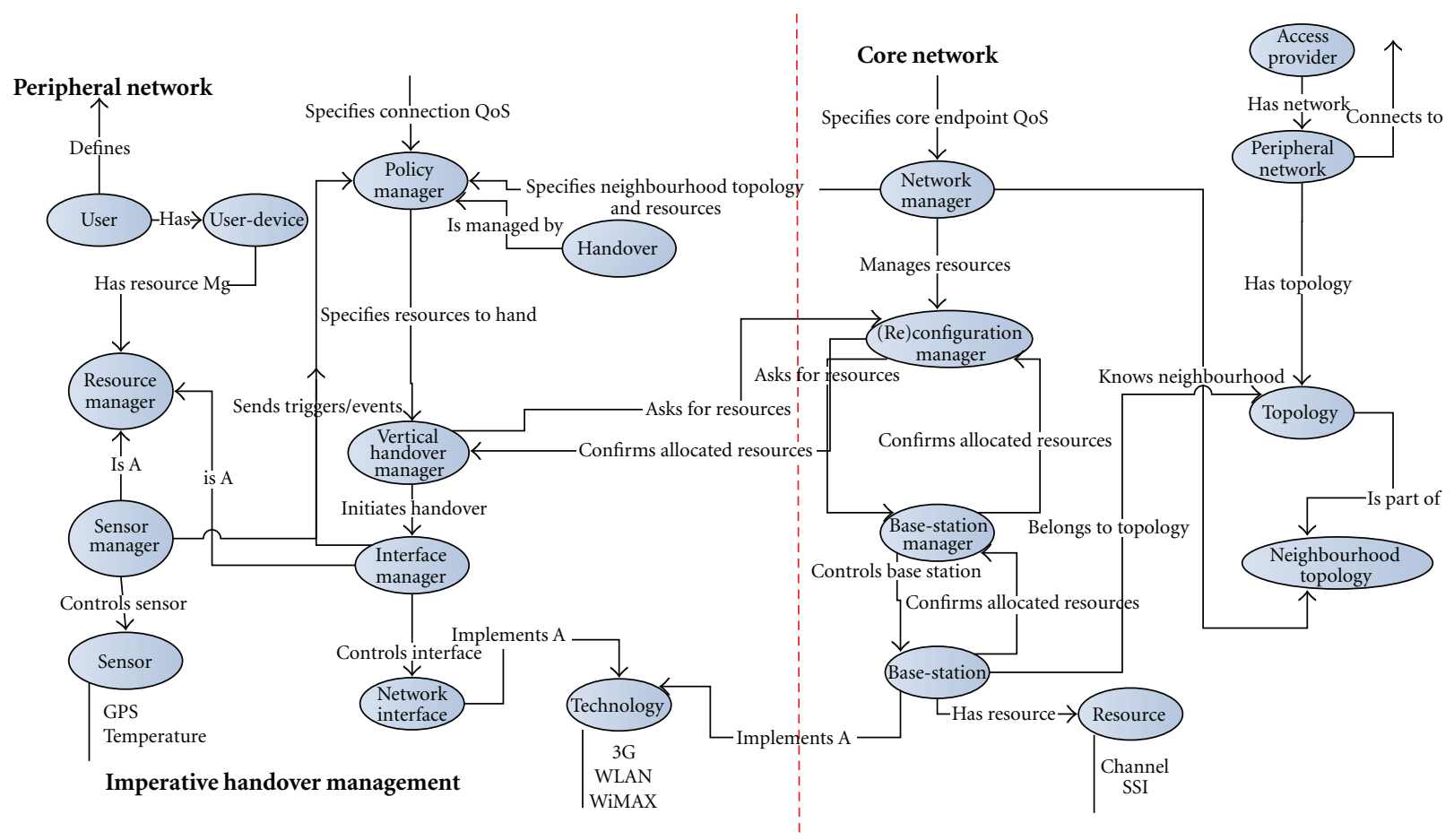

FIGURE 14: An ontology for proactive imperative handover in Y-Comm.

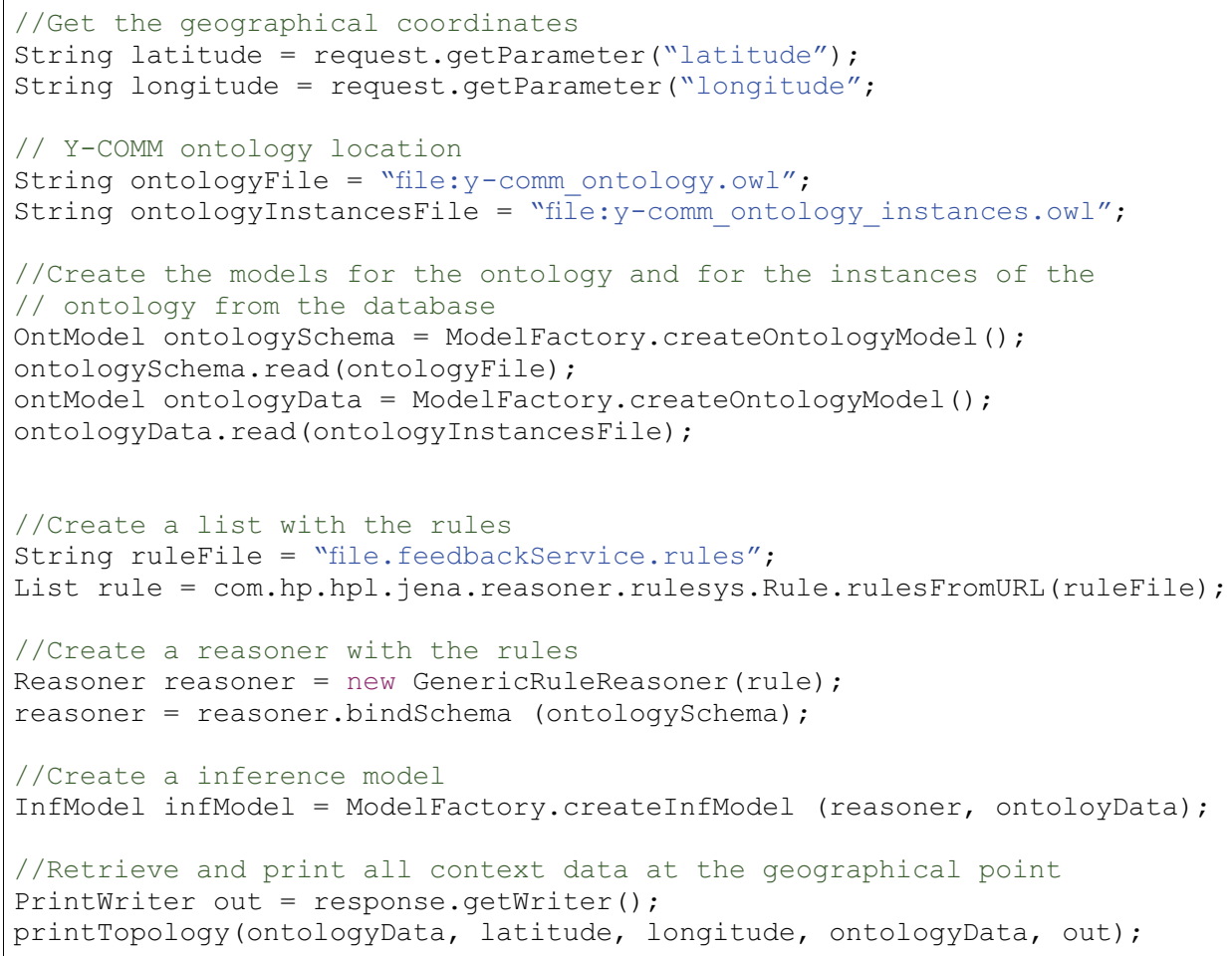

FIGURE 15: Code generated from the Y-Comm ontology. 
Previously stored data is helpful in mobile node movement prediction. The main disadvantage with this approach is it has to depend on a lot of data before making predictions.

9.2. Coverage-Based Prediction. With this technique, handover prediction is based on the knowledge of network coverage which is maintained in a database. So the database contains coverage information instead of records of the movement of the mobile node. Coverage data must be frequently updated for this scheme to work accurately and it is a difficult process maintaining large databases. Soh and Kim [40] proposed a proactive mobility prediction technique. In this technique, horizontal handover time is predicted using the road topology and the location of the mobile node. Recent studies [41] proved that for better network performance calculating the time before handover is worthwhile. The same drawback exists here also in that it is not possible to predict the path and time of mobile node if it takes alternate path instead of the stored one. Cottingham et al. [42] used coverage maps for mapping network coverage. Received signal strength is measured by driving on roads and thus the real-time data is gathered. But the problem is that to gather all the information takes a lot of time which is undesirable.

9.3. Mathematical-Modelling-Based Prediction. In this technique, models and mathematical formulas are used to predict handover conditions. The drawback of this technique is that it can be computationally expensive and the resources of mobile node may be limited. Saleh [43] proposed an algorithm for making location-aided handover decisions. Unwanted vertical handovers are predicted by this algorithm. Another model for predicting unwanted handovers is proposed in [44] and uses a mathematically derived model. Circular WLAN coverage is assumed by this model for calculating the distance between the mobile node and the Access Point, along with received signal strength. This approach fails if the mobile node enters the WLAN network and changes its direction.

This paper shows how the limitations previously mentioned can be overcome. It has shown how to calculate TBVH and how to estimate NDT. It shows how these values can be calculated even when there is a change of direction of the mobile node. This study of proactive handovers has also indicated the need to implement location-based and contextaware services that keep mobile nodes informed of the networks in the local area.

\section{Conclusions and Future Work}

This paper has explored a set of mechanisms which can be used to build a comprehensive framework to support proactive handover in heterogeneous networks. A mathematical framework was first developed and mechanisms to ensure its accuracy using location and contextual information were investigated. Finally, it has been demonstrated how these mechanisms can be integrated into the Y-Comm architecture and how using an ontology for proactive handover provides an implementation path for future systems.
The work presented in this paper was based around the concept of a handover radius. Though this is relevant in most outdoor environments, more sophisticated techniques of defining coverage and the mathematics required to model irregular coverage spaces are being explored. Using the ontological techniques explained above, work has begun to use IEEE 802.21 mechanisms to implement an imperative handover management based on the lower layers of the YComm architecture. This will form the basis of a comprehensive handover framework [37]. Work is also being done to bring up an experimental testbed based on IEEE 802.21. In addition, an investigation is beginning into how to use estimated TBVH and Network Dwell Times to improve channel allocation strategies.

\section{References}

[1] J. Schiller, Mobile Communications, Addison Wesley, 2nd edition, 2003.

[2] J. McNair and F. Zhu, "Vertical handoffs in fourth-generation multinetwork environments," IEEE Wireless Communications, vol. 11, no. 3, pp. 8-15, 2004.

[3] E. Gustafsson and A. Jonsson, "Always best connected," IEEE Wireless Communications, vol. 10, no. 1, pp. 49-55, 2003.

[4] IEEE 802.21 Working Group, "IEEE 802.21: Media Independent Handover Services," http://www.ieee802.org/21.

[5] D. Johnson, C. Perkins, and J. Arkko, RFC 3775 - Mobility Support in IPv6, IETF, 2004.

[6] P. McCann, Mobile IPV6 Fast Handovers for 802.11 Networks, IETF, 2005.

[7] G. Mapp, D. Cottingham, F. Shaikh et al., "An architectural framework for heterogeneous networking," in Proceedings of the International Conference on Wireless Information Networks and Systems (WINSYS '06), pp. 5-10, August 2006.

[8] S. Bansal, A. Lasebae, and R. Comley, "Freedom to choose along-with freedom of mobility," in Proceedings of the International Conference on Information and Communication Technologies: From Theory to Applications (ICTTA '04), p. 191, April 2004.

[9] L. Patanapongpibul, G. Mapp, and A. Hopper, "An end-system approach to mobility management for $4 \mathrm{G}$ networks and its application to thin-client computing," ACM Mobile Computing and Communications Review, vol. 10, no. 3, pp. 13-33, 2006.

[10] D. Cottingham, I. Wassell, and R. Harle, "Performance of IEEE 802.11a in vehicular contexts," in Proceedings of IEEE 65th Vehicular Technology Conference (VTC '07), pp. 854-858, April 2007.

[11] D. Cottingham, Vehicular wireless communications [Ph.D. thesis], Computer Laboratory, University of Cambridge, January 2009.

[12] F. Shaikh, A. Lasebae, and G. Mapp, "Proactive policy management for heterogeneous networks," in Proceedings of the 3rd International Conference on Information and Communication Technologies: From Theory to Applications (ICTTA '08), April 2008.

[13] F. Shaikh, Intelligent proactive handover and QoS management using TBVH in heterogeneous networks [Ph.D. thesis], School of Engineering and Information Sciences, Middlesex University, January 2010.

[14] W. Chen, J. Liu, and H. Huang, "Implementation and performance study of IEEE 802.21 in integrated IEEE 802.11/802.16e 
networks," in Proceedings of the 10th International Conference on Parallel and Distributed Systems, 2004.

[15] V. Abhayawardhana, I. Wassell, D. Crosby, M. Sellars, and M. Brown, "Comparison of empirical propagation path loss models for fixed wireless access systems," in Proceedings of IEEE 61st Vehicular Technology Conference (VTC '05), pp. 73-77, Springer, June 2005.

[16] "Radio theory and link planning for Wireless LAN (WLAN)," http://www.swisswireless.org/wlan_calc_en.html.

[17] G. Mapp, F. Shaikh, M. Aiash, R. Vanni, M. Augusto and E. Moreira, "Exploring efficient imperative handover mechanisms for heterogeneous networks," in Proceedings of the International Symposium of Emerging Ubiquitous and Persuasive Systems, Indianapolis, Ind, USA, August 2009.

[18] Wikipedia, "Article on WiFi," http://en.wikipedia.org/wiki/ Wi-Fi.

[19] J. Medisetty, Exploring coverage areas to facilitate vertical proactive handovers [M. Sc. thesis], School of Engineering and Information Sciences, Middlesex University, November 2011.

[20] S. Maniyar, Characterising network attachment points to facilitate proactive vertical handover [M. Sc. thesis], School of Engineering and Information Sciences, Middlesex University, November 2011.

[21] N. Chinnam, Exploring different propagation models for wireless networks for different environments [M. Sc. thesis], School of Engineering and Information Sciences, Middlesex University, February 2012.

[22] M. Braga, "Cell phone reception explained: what the bars mean," July 2010, http://www.tested.com/news/cell-phonereception-explained-what-the-bars-mean/510/.

[23] V. Andrei, E. C. Popovici, O. Fratu, and S. V. Halunga, "Development of an IEEE 802.21 media independent information service," in Proceedings of the 17th IEEE International Conference on Automation, Quality and Testing, Robotics (AQTR '10), pp. 123-128, May 2010.

[24] D. Dave, Local network information for proactive handovers [ $M$. Sc. thesis], School of Engineering and Information Sciences, Middlesex University, August 2010.

[25] D. Cottingham and P. Vidales, "Is latency the real enemy in next generation networks?" in Proceedings of the 1st International Workshop on Convergence of Hetergeneous Wireless Networks, July 2005.

[26] R. R. F. Lopes, B. J. van Beijnum, and E. D. S. Moreira, "Towards a feasible social-based methodology to manage wireless connectivity context data," in Proceedings of IEEE 7th International Conference on Wireless and Mobile Computing, Networking and Communications (WiMob '11), pp. 237-244, Shanghai, China, October 2011.

[27] R. R. F. Lopes, B. J. Van Beijnum, and E. D. S. Moreira, "Managing wireless IP-connectivity experiences as mobile social media," in Proceedings of the International Congress on Ultra Modern Telecommunications and Control Systems and Workshops (ICUMT'10), pp. 154-161, October 2010.

[28] E. D. S. Moreira, J. Crowcroft, G. Mapp, D. Cottingham, P. Hui, and R. Vanni, "Exploiting contextual handover information for versatile services in NGN environments," in Proceeings of the 2nd International Conference on Digital Information Management (ICDIM '07), October 2007.

[29] U. Horn, C. Prehofer, H. Karl et al., "Ambient networks: an architecture for communication networks beyond 3G," IEEE Wireless Communications, vol. 11, no. 2, pp. 14-22, 2004.
[30] M. Kuroda, M. Yoshida, R. Ono, S. Kiyomoto, and T. Tanaka, "Secure service and network framework for mobile ethernet," Wireless Personal Communications, vol. 29, no. 3-4, pp. 161190, 2004.

[31] M. Aiash, G. Mapp, A. Lasebae et al., "A survey of potential architectures for communication in heterogeneous networks," in Proceedings of the Wireless Telecommunications Symposium (WTS '12), April 2012.

[32] Y-Comm Web Page, http://www.mdx.ac.uk/research/areas/ software/ycomm_research.aspx.

[33] G. Mapp, F. Shaikh, J. Crowcroft, D. Cottingham, and J. Baliosian, "Y-Comm: a global architecture for heterogeneous networking," in Proceedings of the 3rd Annual International Wireless Internet Conference (WICON '07), October 2007.

[34] J. der Merwe and I. Leslie, "Switchlets and dynamic virtual ATM networks," in Proceedings of the Integrated Network Management $V, 1997$.

[35] M. Aiash, G. Mapp, and A. Lasebae, "Security and QoS integration for protecting service providers in hterogeneous environments," IAENG International Journal of Computer Science, vol. 38, no. 4, pp. 384-393.

[36] M. Aiash, G. Mapp, A. Lasebae, J. Loo, and R. Phan, "A formally verified AKA protocol for vertical handover in heterogeneous environments using Casper/FDR," EURASIP Journal on Wireless Communincations and Networking, 2012. In press, http://jwcn.eurasipjournals.com/content/2012/1/57/.

[37] R. Vanni, K. Branco, E. Moreira, and G. Mapp, "Information for handover management in heterogeneous networks: data representation, languages and integrated platforms," in Proceedings of the 7th CONTECSI-International Conference on Information Systems and Technology Management, pp. 1-13, Sao Paulo, Brazil, May 2010.

[38] P. Vidales, G. Mapp, F. Stajano, J. Crowcroft, and C. J. Bernardos, "A practical approach for $4 \mathrm{G}$ systems: deployment of overlay networks," in Proceedings of the 1st International Conference on Testbeds and Research Infrastructures for the Development of Networks and Communities (Tridentcom '05), pp. 172181, IEEE Computer Society, February 2005.

[39] P. Vidales, R. Chakravorty, and C. Policroniades, "PROTON: a policy-based solution for future $4 \mathrm{G}$ devices," in Proceedings of the 5th IEEE International Workshop on Policies for Distributed Systems and Networks (POLICY '04), pp. 219-222, June 2004.

[40] W. Soh and H. S. Kim, "Dynamic bandwidth reservation in cellular networks using road topology based mobility predictions," in Proceedings of IEEE 23rd Annual Joint Conference of the IEEE Computer and Communications Societies (INFOCOM '04), pp. 2766-2777, March 2004.

[41] F. Shaikh, G. Mapp, and A. Lasebae, "Proactive policy management using TBVH mechanism in heterogeneous networks," in Proceedings of the International Conference on Next Generation Mobile Applications, Services and Technologies (NGMAST '07), pp. 151-157, September 2007.

[42] D. Cottingham, R. Harle, and A. Hopper, "Constructing accurate, space-efficient, wireless coverage maps for vehicular contexts," in Proceedings of the 4th International Wireless Internet Conference (ISTWICON '08), November 2008.

[43] A. Saleh, A location-aided decision algorithm for handover across heterogeneous wireless overlay networks [M.Sc. thesis], Virginia Polytechnique Institute and State University, 2004.

[44] X. Yan, N. Mani, and Y. Cekercioglu, "A traveling distance prediction based method to minimize unnecessary handovers from cellular networks to WLANs," IEEE Communications Letters, vol. 12, no. 1, pp. 14-16, 2008. 

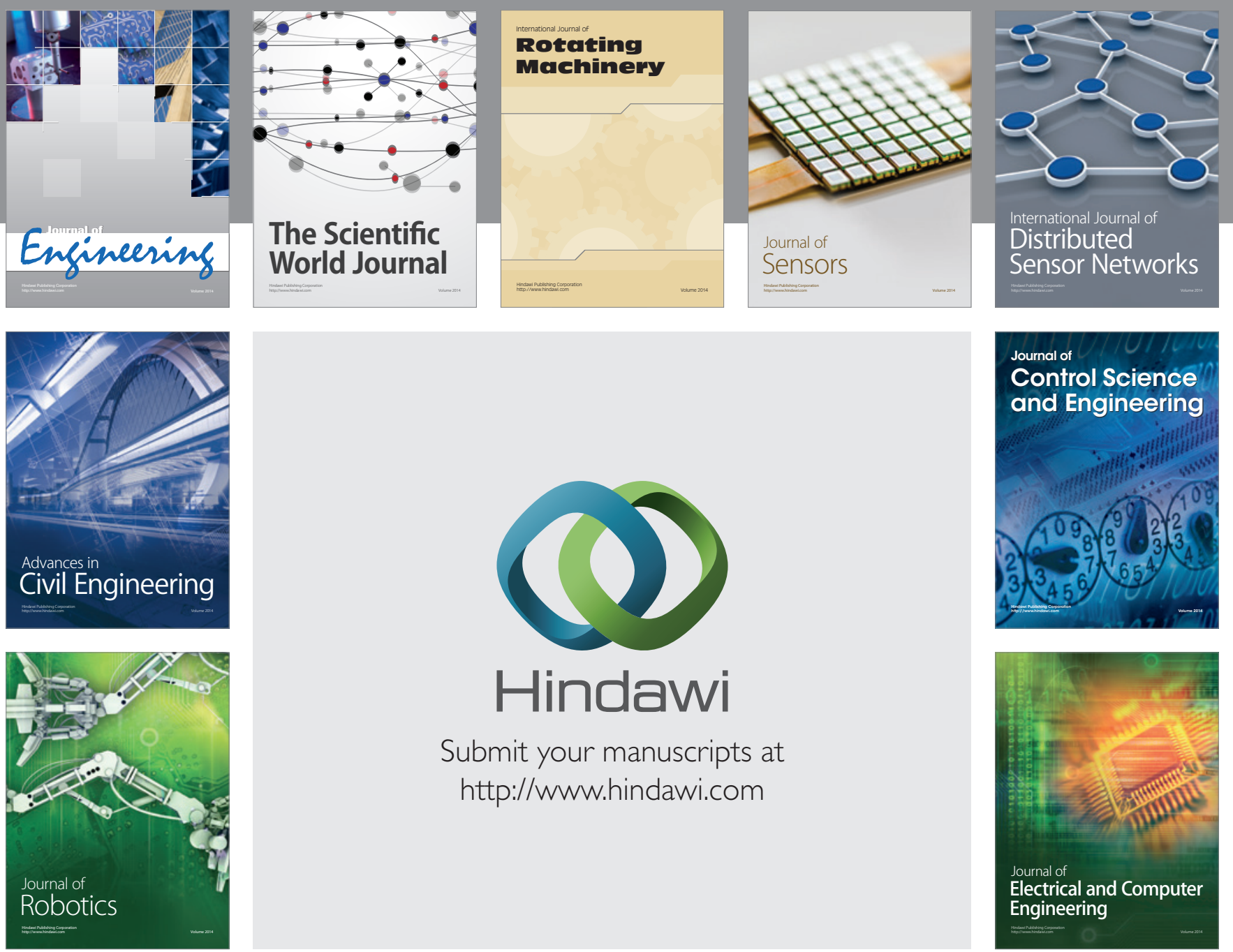

Submit your manuscripts at

http://www.hindawi.com
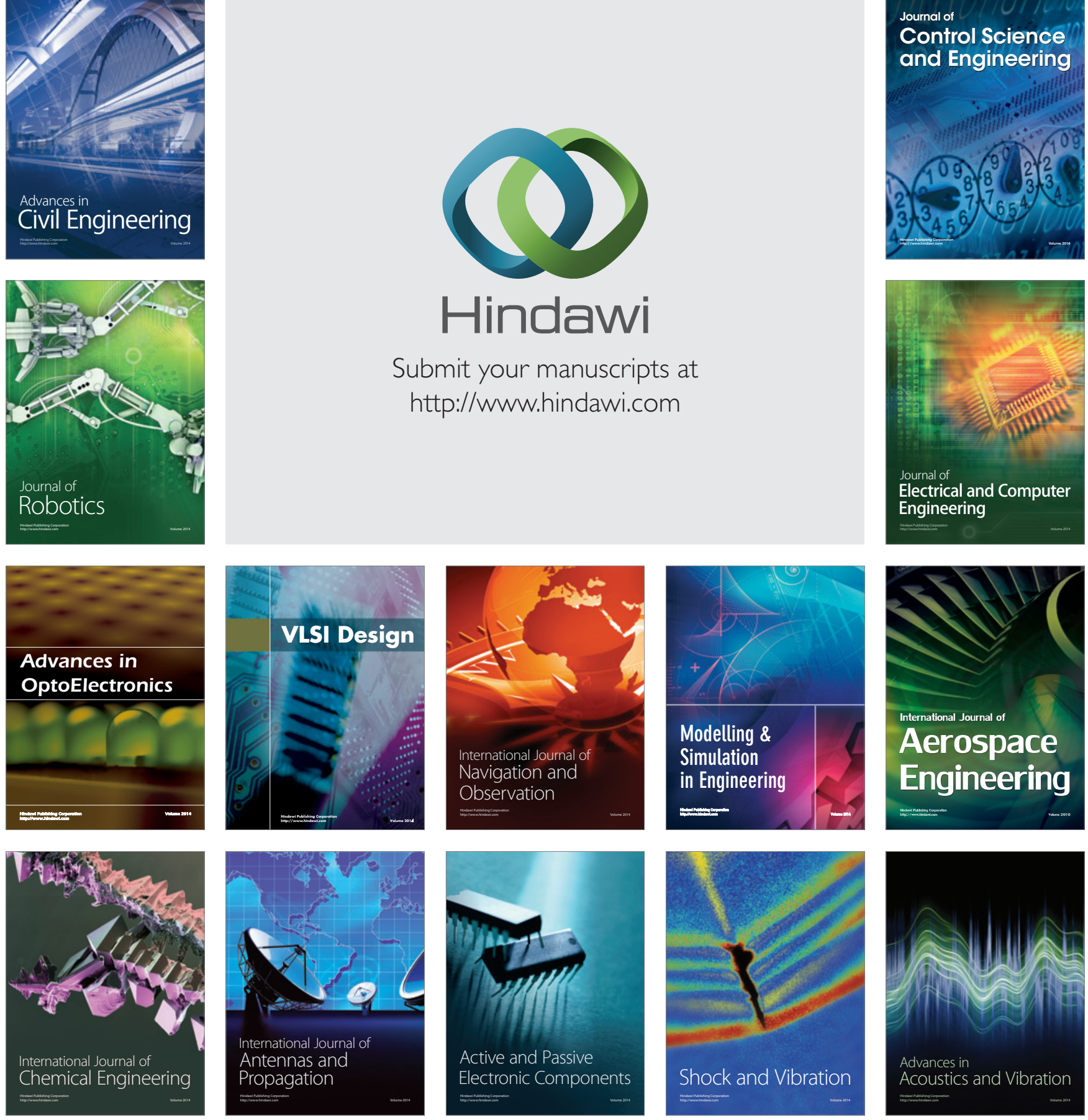